\title{
DNA sensors, crucial receptors to resist pathogens, are deregulated in colorectal cancer and associated with initiation and progression of the disease
}

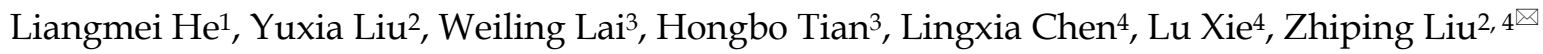 \\ 1. Department of Gastroenterology, The First Affiliated Hospital of Gannan Medical University; \\ 2. Center for Immunology, Key Laboratory of Prevention and Treatment of Cardiovascular and Cerebrovascular Diseases, Ministry of Education, Gannan \\ Medical University \\ 3. Gannan Medical University; \\ 4. School of Basic Medicine, Gannan Medical University, Ganzhou, Jiangxi, 341000 China. \\ $\square$ Corresponding author: Zhiping Liu, School of Basic Medicine, Gannan Medical University, Ganzhou, Jiangxi, 341000, China. Tel: (86) 13879793709; Email: \\ Zhiping.Liu@gmu.edu.cn
}

() The author(s). This is an open access article distributed under the terms of the Creative Commons Attribution License (https://creativecommons.org/licenses/by/4.0/). See http://ivyspring.com/terms for full terms and conditions.

Received: 2019.02.17; Accepted: 2019.10.20; Published: 2020.01.01

\begin{abstract}
Background: DNA sensors are innate immune receptors that detect intracellular endogenous or exogenous DNA. They are critical to trigger immune response against DNA viral and intracellular bacterial infection, and are involved in inflammatory diseases and tumorigenesis. Recent accumulating evidences indicated that DNA sensors are also crucial for controlling the development of colorectal cancer (CRC). However, a systematic study on the expression profile of DNA sensors in CRC and their clinical significance are still lacking.

Methods: We investigated the expression profile of DNA sensors in CRC and their clinical significance by taking advantage of clinical CRC samples, mouse AOM/DSS treatment model, and Oncomine $₫$ bioinformatics platform.

Results: Our study identified that the expression of DNA sensors, including AIM2, DAl, as well as inflammasome molecules ASCIIL-18, TLR9 and adaptor MyD88, and DDX60 decreased in human CRC, whereas the expression of DHX9, DHX36, and DDX4l significantly increased. Among them, the expression of AIM2/ASCIIL-18, MyD88, DAI, DHX36, and DDX60 were associated with cancer stages. In addition, we also performed correlation analysis between DNA sensors and their main signaling molecules to explore the possible mechanisms. The results showed that there were positive correlations between AIM2 and ASC/IL-18, DHX9 and MAVS, and TLR9 and MyD88 expression. In addition, the gene expression patterns of some DNA sensors were confirmed by Western-blot analysis.

Conclusions: Our study revealed that the expression of multiple DNA sensors was deregulated in $C R C$ and might be involved in tumor development. More importantly, the study identified that, among all these DNA sensors, AIM2, DAl, and DDX60 could be potentially critical for diagnosis, prognosis, and therapy of CRC and deserve further investigation.
\end{abstract}

Key words: DNA sensors, expression profile, cancer, cancer stages

\section{Introduction}

Colorectal cancer (CRC) is one of the major diseases that are associated with cancer-related death throughout the world. Over 1.8 million new colorectal cancer cases and 881,000 deaths are estimated to occur in 2018 [1]. Although the incidence and mortality of CRC have declined in some developed countries in recent years, they still show an upward trend in some developing or under-developed countries [2]. When 
most of the patients are diagnosed with CRC, they have been already in the advanced stage. Therefore, the combination of surgery treatment and postoperative chemotherapy are often required and the treatment effect is usually not optimal. All these factors have brought a heavy burden to the society and economic health. Therefore, exploring new molecular mechanisms of CRC development may provide new ideas and targets for the diagnosis and treatment of CRC.

The onset of CRC is a multistep, multifactorial, and polygenic process, but chronic inflammation is well recognized as a risk factor for CRC. Clinically, inflammatory bowel disease (IBD) patients are more likely to develop into CRC than healthy people [3]. The IBD mouse model also supports the notion that inflammation can promote the development of CRC [4]. The innate immune receptors, pattern recognition receptors (PRRs), are the key factors to trigger the inflammatory response [5]. In addition to regulating inflammation development, some PRRs also play an important role in the process of cell proliferation and apoptosis [6]. These receptors mainly include Toll like receptors (TLRs), Nod-like receptor (NLRs), RIG-I like receptor (RLRs), AIM2 like receptor (ALRs), and C-type lectin receptor (CLRs). After activation by the pathogen-associated molecular patterns (PAMPs) or danger-associated molecular patterns (DAMPs), PRRs can induce self-activation and cytokine release, and further activate the adaptive immune cells, so as to mount potent antiviral immune response [7] .

The DNA sensors are one of the major types of innate immune receptors. Most of the DNA sensors are present and functional in the cytoplasm [8] [9]. DNA sensors mainly include ALRs, such as Absent in melanoma 2 (AIM2) and Interferon gamma inducible protein 16 (IFI16), cGAMP synthase (cGAS), Z-DNA binding protein $1(\mathrm{ZBP}-1$ or DAI), Toll like receptor 9 (TLR9) and helicase family members, such as DEAH-Box Helicase 9 (DHX9), DEAD-box helicase 41(DDX41), and DEAD-box helicase 60 (DDX60) [10]. They can detect abnormal intracellular endogenous or exogenous nucleic acids, and then induce activation of the inflammasome or the production of type I interferons (type I IFNs) and other inflammatory cytokine through a series of downstream adaptor molecules, thus effectively defensing host against intracellular bacterial and viral infection [11].

However, recent accumulating evidences showed that DNA sensors, such as AIM2, TLR9, and adaptor molecule STING, are important not only for host defense against pathogens, but also for controlling the development of colitis and CRC [9] [12] [13] [14].
AIM2
double-stranded DNA

(dsDNA) to mediate the assemble of a multi-protein complex, inflammasome, through binding to apoptosis-associated speck-like protein containing a CARD (ASC) and Caspase-1, which then cleaves pro-IL-1 $\beta$ and pro-IL-18 into mature forms [15].

However, studies have shown that AIM2 inhibits the occurrence of CRC by regulating intestinal epithelial cells via an inflammasome-independent manner [16] [17] [18]. STING is a cytoplasmic receptor for cyclic dinucleotides and an important adaptor protein of other DNA receptors, such as DAI, cGAS, DDX41, and IFI16 [19] [20] [21] [22]. These upstream DNA receptors transmit signals through STING and modulate the production of type I IFNs. STING inhibits the development of CRC by inhibiting intestinal inflammation and stimulating the production of type I IFNs [23] [24] [25].

In addition, studies have shown other DNA sensors, such as DHX9, DDX41, and DDX60, also play important roles in the development of tumors [26] [27] [28]. Therefore, we hypothesize that DNA sensors may play important roles in the development of colitis and CRC. However, in general, most of the signaling pathways of these DNA sensors and their roles in the development of CRC remain largely unknown. Furthermore, whether these DNA sensors in CRC tissues and normal tissues are differentially expressed, and whether their expressions are associated with clinical stages, grades, and prognosis, are yet to be investigated.

In this study, the expression AIM2, IFI16, DAI, TLR9, cGAS, DHX9, DHX36, DDX41, DDX60 of DNA sensors as well as inflammasome molecules ASC/IL-18, TLR9 and adaptor MyD88, and adaptor molecule STING in human were examined. We also took the advantage of Oncomine data platform to further analyze their correlation with clinical cancer stages. Oncomine is a large bioinformatics integration platform, which is designed to collect, standardize, analyze and communicate transcription data to biomedical researchers [29]. Finally, we used early-stage mouse CRC models to investigate whether DNA sensors are involved in the formation of CRC. This study can not only benefit us for better understanding on the expression profile of DNA sensors in CRC, but also provide a theoretical basis for the diagnosis, prognosis, and treatment of CRC.

\section{Materials and Methods}

\section{Clinical sample collection}

CRC tissues and adjacent tissues were collected from Department of Gastrointestinal Surgery in the First-Affiliated Hospital of Gannan Medical University (Ganzhou, Jiangxi, China) (Supplemental 
Table 1). Patients who were newly diagnosed with CRC based on the clinicopathological criteria were included. Patients who received preoperative chemo-, radio- or immunotherapy were excluded. Upon resected, the cancer and the adjacent normal tissues (above $3 \mathrm{~cm}$ away from cancer tissue) were stored in liquid nitrogen for subsequent qRT-PCR analysis.

\section{AOM (Azoxymethane) /DSS (Dextran sodium sulfate) treatment}

AOM and DSS treatment by one dose of carcinogen AOM injection and three rounds of DSS treatment in drinking water is a well-established colitis-associated CRC animal model [30]. In order to study the causes, not the results, of CRC development, AOM and DSS treatment by one dose of AOM injection and one round of DSS treatment (14 days after AOM treatment) is often used to study pre-cancerous mucosal gene expression changes [17, 31-33].

The 6-8 weeks old C57 BL/6 mice were divided into the experimental group and the control group. The experimental group received intraperitoneal injection of AOM (Sigma, USA, 10ug/g body weight), and the control group was injected with PBS. Five days after AOM injection, the mice in experimental group were fed up with 3\% DSS water (MP Biologicals, USA), and the control group was fed up with regular drinking water. After 6 days, the 3\% DSS water was replaced by regular drinking water for 3 days, and then some mice were sacrificed (day 14). The colorectal tissues were collected for further analysis.

\section{qRT-PCR}

The total RNA of tissues was extracted using Trizol reagent (Invitrogen, USA). The cDNA was synthesized by M-MLV First Strand Kit (Invitrogen, USA). Then the Real-time PCR of DNA sensors were performed in Bio-rad Real-Time PCR System with the Sybergreen Master Mix (Invitrogen, USA). The primer sequences of DNA sensors were shown (Supplemental Table 2, 3). The $\beta$-actin was chosen as a reference gene. The relative expression of target genes in cancer and the control groups was expressed as $2-\Delta \Delta C T$.

\section{Data collection from Oncomine}

Oncomine (https://www.oncomine.org) is the largest cancer microarray integrated platform worldwide, which aims to dig for cancer genetic information. So far, 729 gene expression datasets, more than 90000 cancer tissues and normal tissue samples were collected in this platform. In this study, the TCGA database in the Oncomine platform was chosen to study the expression profile of the DNA sensors (Analysis Type: Cancer vs. Normal Analysis; Cancer Type: Colorectal Cancer; Data Type: mRNA; Sample Type: Clinical Specimen; Genes: every one DNA sensors; Dataset name: TCGA Colorectal, Threshold fold change $\geq 1.5, \mathrm{p}$ value $\geq$ IE-4, Gene Rank in the top 10).

\section{Western-blot analysis}

Proteins were extracted from colorectal tissues by RIPA lysis buffer with proteinase inhibitors. Samples were resolved in SDS-PAGE and transferred onto NC membranes. Membranes were blocked in 5\% milk for 1 hour and incubated in primary antibodies overnight at 4 degree. Membranes then were incubated with HRP-conjugated secondary antibody for 1 hour, and proteins were visualized using ECL substrates. The primary antibodies include STING (13647, Cell Signaling), IFI16 (ab55328, Abcam), DAI (sc-271483, Santa Cruz), DDX41 (15076, Cell Signaling), DHX9 (12266, Affinity), DHX36 (ab70269, Abcam), DDX60 (ab139807, Abcam) and $\beta$-ACTIN (A5441, Sigma).

\section{Data analysis}

All data were analyzed in statistical software Prism 7.0. The quantitative data were represented by Mean \pm SEM. As for qRT-PCR results, the relative expression of target genes in cancer and the control groups was expressed as 2- $\Delta \Delta \mathrm{CT}$. The expression differences of DNA sensors between human CRC tissues or AOM/DSS treated mouse tissues and the controls were analyzed by paired $t$ test or unpaired $t$ test, respectively. Quantitative data between groups were analyzed by ANOVA (variance). When the correlation between molecules was analyzed, the two sets of data were sequenced according to the order of the samples, and the correlation between the molecules was analyzed by multivariate linear regression analysis (Liner, regression). $p<0.05$ was considered statistically significant. ${ }^{*} p<0.05,{ }^{* *} p<0.01$, *** $p<0.001$.

\section{Results}

\section{The expression profile of AIM2 and inflammasome-associated molecules in CRC}

AIM2 is a cytoplasmic DNA sensor that mainly functions via inflammasome. An European study showed that low level of AIM2 in colon tissues of CRC patients was associated with poorer prognosis [34]. However, genetic and epigenetic difference among regions and racial may exist [35]. Recent study had shown that AIM2 and adjacent normal controls were not significantly different in Chinese CRC patients [36] . qRT-PCR results of our CRC samples showed that the expression of AIM2 significantly decreased in 
CRC tissues, in comparison with the controls (Figure 1A). The sample content and quality difference may also contribute to this discrepancy. More studies are required to investigate the AIM2 expression in Chinese CRC patients.

To further study whether the expression changes of AIM2 in CRC were a universal phenomenon, we carried out a large sample analysis by taking advantage of Oncomine platform. Oncomine is the largest integration bioinformatics platform for microarrays, where the data were standardized uniformly [29]. We chose the TCGA sub-database, including data from 215 CRC samples and 22 healthy samples, to further analyze the expression profile of DNA sensors. Consistently, we found that the expression of AIM2 was significantly reduced in CRC in TCGA database (Figure 1C). Similarly, the expression of AIM2 inflammasome downstream molecules, ASC and IL-18, decreased in CRC (Figure 1D, E). Subsequently, we analyzed the expression of AIM2, ASC, and IL-18 in different stages of CRC. However, in contrast to $A S C$ and $I L-18$, the expression of AIM2 in different stages of CRC was not significantly different from healthy control group

(Figure 2A, B, C). IL-18 displayed statistically different expression levels between healthy control and various CRC stages (Figure 2C), whereas only the ASC expression in CRC stage I was significantly different from health control (Figure 2B).

Previous in vivo studies found that the preventive effect of AIM2 in mouse CRC is independent of inflammasome activation and may be associated with the inhibition of AKT proliferation signaling pathway [16, 17]. A recent in vitro study also found that up-regulation of AIM2 in CRC cell line HCT116 promoted cell apoptosis that may be associate with decreased AKT level [18]. However, the formation and progression of tumor is determined by the complicated interaction between tumor and tumor microenvironments. Whether the preventive role of AIM2 in CRC is associated with inflammasome is still controversial. Our current results showed a positive correlation between AIM2 and inflammasomeassociated molecules ASC and $I L-18$ in CRC (Figure 2D, E). These results indicate that AIM2 in CRC samples may function in either inflammation-dependent or inflammationindependent manners.

On the other hand, the gene expression change may reflect either the causes of tumor or the results of tumor formation. To further investigate whether AIM2 is involved in early stage of CRC formation, we employed the classical mouse inflammationassociated CRC model induced by AOM and DSS treatment. The mice were sacrificed at day 14 after a dose of AOM injection and one round of DSS treatment, which is a critical time point often used to study factors to induce the development of CRC [17] [31-33]. The body weight loss and shortening of the colon are two of the major indexes of inflammation [30]. The AOM/DSS-treated 
mice lost more body weight, displayed shorter colon lengths over the process of AOM/DSS treatment. In addition, H\&E staining results showed that the normal colonic mucosa structure of the experimental group was disrupted [37]. Subsequent qRT-PCR results showed that the colonic expression of Aim2 was significantly downregulated in AOM/DSS-treated group in comparison with control groups (Figure 1B). In summary, our study showed that AIM2 and AIM2 inflammasome downstream molecules were downregulated in CRC samples,
A

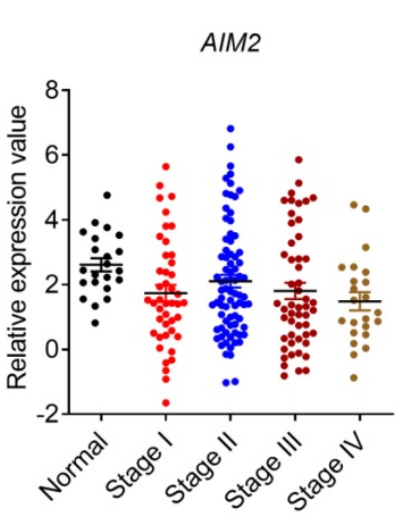

C

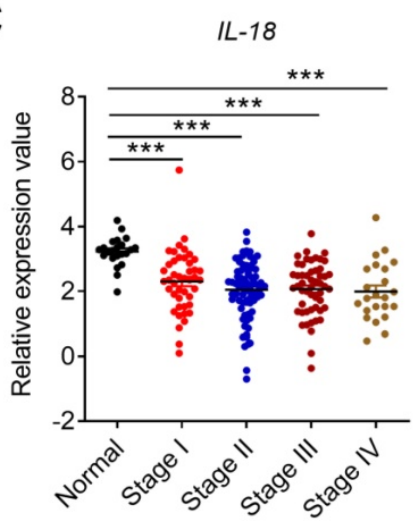

$\mathbf{E}$

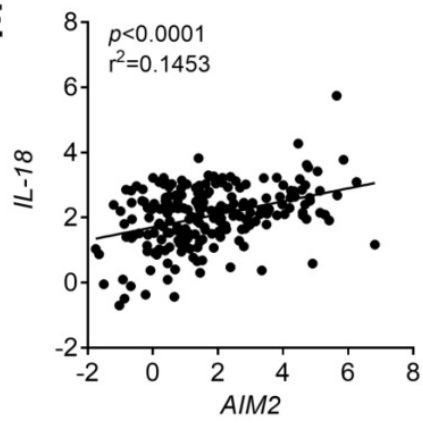

B

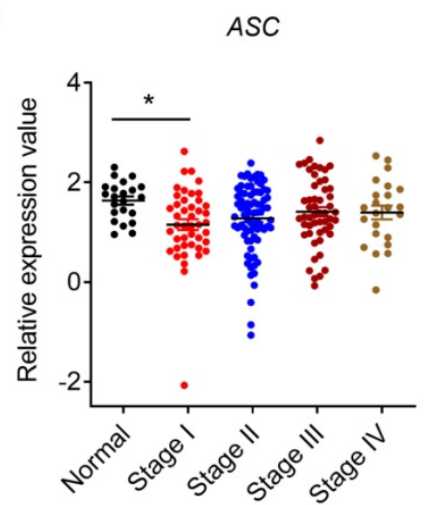

D

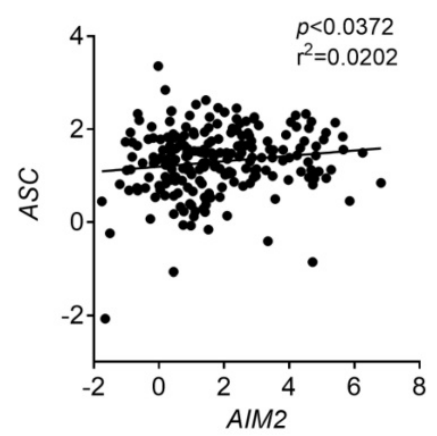

indicating that they may be involved in CRC formation and progression.

\section{The expression profile of STING signaling-associated molecules in CRC}

STING is crucial for the production of type I IFNs and activation of $\mathrm{CD}^{+} \mathrm{T}$ cells $[38,39]$. On one hand, STING can be activated by cyclic-dinucleotides (CDNs) that is secreted by bacteria or synthesized by cyclic GMP-AMP synthase (cGAS) [40] . On the other hand, STING is also the adaptor for some other DNA sensors include DDX41, IFI16, and DAI [27] [22]. A previous study indicated that Sting-mice were more susceptible to AOM/DSSinduced CRC [23]. In addition, functional defects and variant in STING signaling were frequently observed in human CRC cell lines [41]. Our study found that the expression of STING displayed no significant change in clinical CRC samples, samples from AOM/DSS treated mice, or TCGA database, and in different stages of CRC (Figure 3A, B, $C$, Figure 4A). These results were different from another recent study, which showed that STING expression was significantly lower in Chinese CRC patients and there were a dysregulation of the cGAS/IFI16STING-TBK1-IFN $\beta$ pathway [42].

Interestingly, the expression level of cGAS was undetectable in our CRC samples (Data not shown), which may be partially associated with the methylation of cGAS in CRC cells [41]. Furthermore, the methylation of cGAS in some of our CRC samples might also affect the STING expression, thus leading to the discrepancy between our study and previous studies.

Although IFI16 belongs to the member of ALRs, it mainly functions via STING signaling pathway [22]. IFI16 plays an important role in virus restriction, however, its role in tumor setting is not well studied. No significant expression difference of IFI16 were observed in our clinical CRC samples (Figure 3D). In addition, we examined the expression levels of p204, a homolog to human IFI16, in colon tissues of 14 days after AOM/DSS treatment in mice. The results of p204 also showed no significant expression changes in our mouse samples (Figure 3E). But its expression levels decreased in TCGA database (Figure 3F). However, no correlation between IFI16 expression level and cancer stages was identified (Figure 4B). The role of DAI in tumor setting, especially
Figure 2. Correlation analysis between the expression of AIM2 inflammasome molecules and CRC stages, and correlation between AIM2 and inflammasome retrieved from the TCGA microarray database in Oncomine $\AA$ platform, and were grouped by stages or patient IDs for further analysis. (A-C) Correlation analysis between the expression of AIM2, inflammasome molecules (ASC and IL-I8) and cancer stages were shown. (D-E) Correlation between the expression of AIM2 and ASC/IL-I8. $n=22$ for health normal control, 44 for stage I, 78 for stage II, 52 for stage III, and 23 for stage IV. Data were expressed as mean \pm SEM, Log2 median-centered ratio expression. $* p<0.05 ; * * * p<0.001$. molecules. The expression data of AIM2 and inflammasome molecules, ASC and IL-I8, were 
in $\mathrm{CRC}$, is also unclear. We found that DAI expression decreased in the early stage of tissues from AOM/DSS treated mice and TCGA database (Figure $3 \mathbf{H}, \mathbf{I})$, whereas no significant change was observed in our clinical samples (Figure 3G). Furthermore, DAI expression levels were associated with cancer stages (Figure 4C).
A

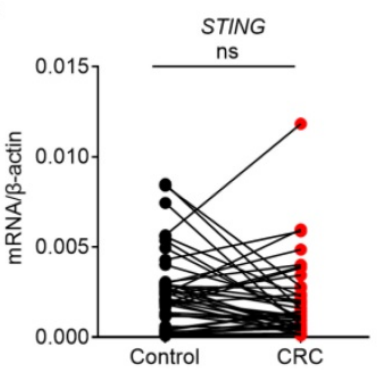

D

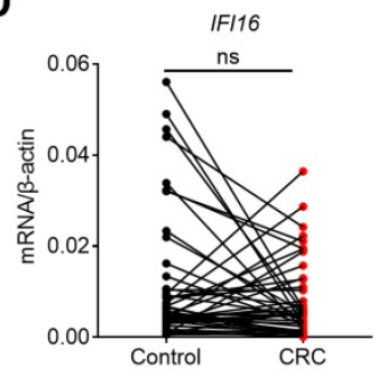

G

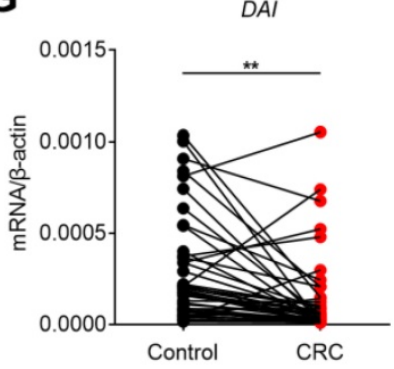

$\mathbf{J}$

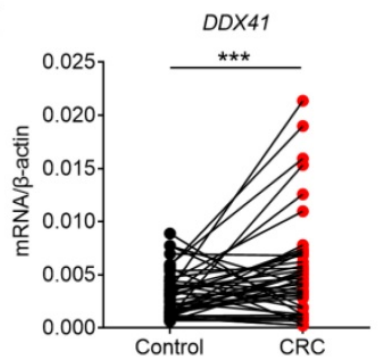

B

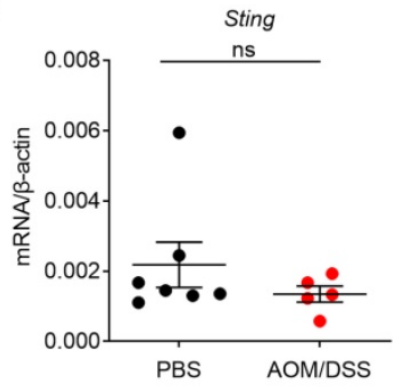

$\mathbf{E}$

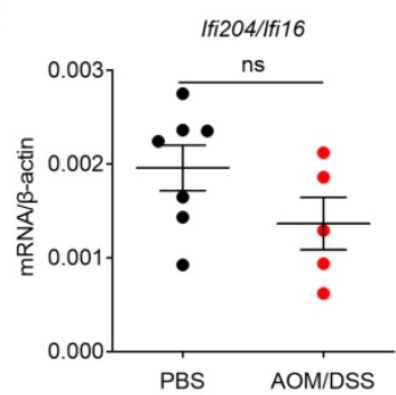

H
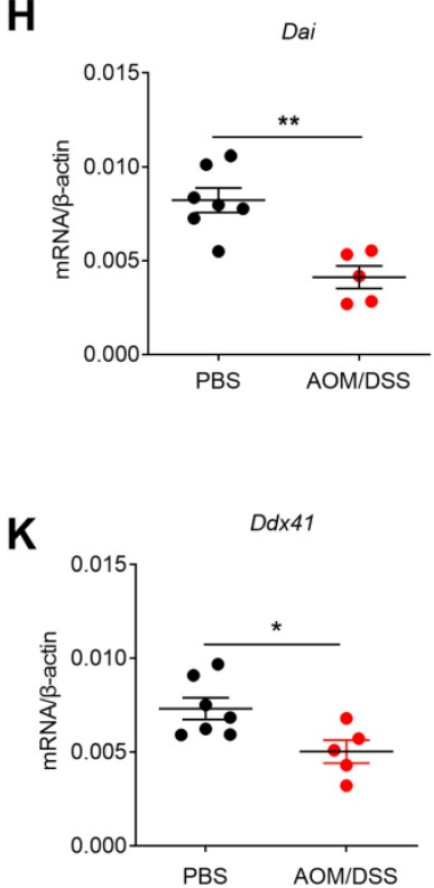

C

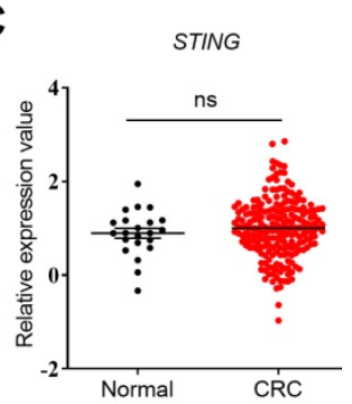

$\mathbf{F}$

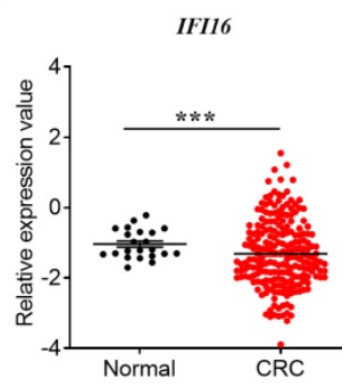

I

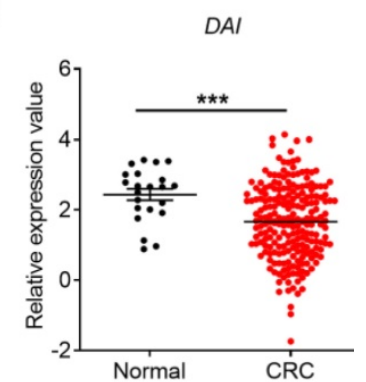

$\mathbf{L}$

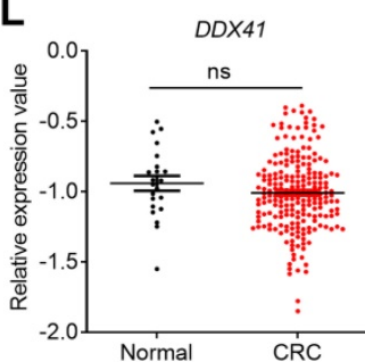

Figure 3. The expression profile of STING signaling pathway-associated molecules in human and mouse CRC tissues and TCGA database. RNA was extracted from cancer and matched peri-carcinomatous tissues of CRC patients, as well as tissues from AOM/DSS treated mice and reverse-transcribed into cDNA. Then the gene expression levels of STING signaling pathway-associated molecules were determined by quantitative PCR. The data from the same patient were connected by straight lines. The data of the expression of STING signaling pathway-associated molecules were retrieved from the TCGA microarray database in Oncomine $®$ platform. Expression of STING (A, B, C), IFII 6 (D, E, F), DAI (G, H, I) and DDX4I (J, K, L) in human CRC tissues, tissues from AOM/DSS treated mice, and TCGA database. Control: matched peri-carcinomatous tissues, $n=44$ for clinical samples, data represent two independent experiments; $n=7$ for PBS-treated mice and $n=5$ for AOM/DSS- treated mice; $n=22$ for health normal controls and $n=215$ for CRC group. $* p<0.05, * * p<0.01$, $* * * p<0.001 ;$ NS: Not significantly different. 
As a member of helicase family, DDX41 detects dsDNA and signals via STING to mediate the production of Type I IFNs. However, the function of DDX41 is much more complicated and was also reported to participate in RNA metabolism. A recent study found that DDX41 mutation was frequently observed in human hematological malignancy [27]. However, the role of DDX41 in solid tumor is still unknown. Our results showed that DDX41 expression in human CRC was much higher than the control groups (Figure $3 \mathbf{J}$ ) but the association with tumor stages was not identified (Figure 3H). DDX41 expression levels had no significant changes in TCGA database (Figure 4D). In addition, $D d x 41$ expression
A

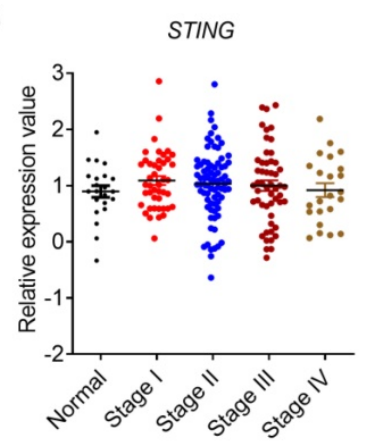

C

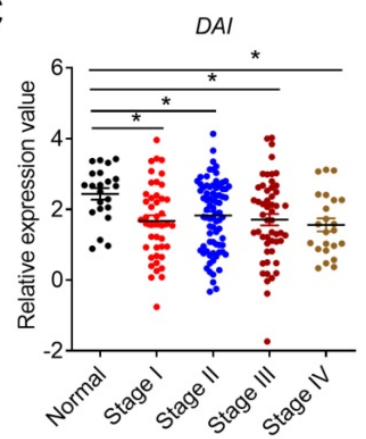

E

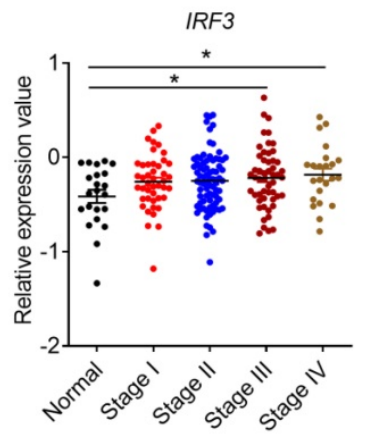

B

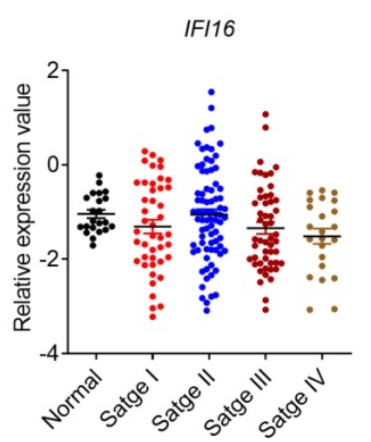

D

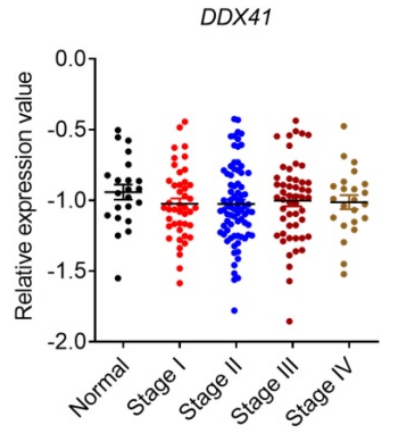

decreased in AOM/DSS treated mice (Figure 3K). The discrepancy between the mouse model and human CRC could be possibly due to the time point difference.

Given that STING-TBK1-IRF3 signaling pathway is important for the production of type I IFNs and $\mathrm{CD}^{+} \mathrm{T}$ cell-mediated anti-tumor response, the expression of IRF3 in CRC was also analyzed in TCGA database. We found that the mRNA level of IRF3 increased in CRC tissues and was associated with tumor stages (Figure 4E).

\section{The expression profile of helicases family members in CRC}

Other DNA sensors in helicase family included DHX9, DHX36, and DDX60. A study suggested that they are indispensable for type I IFN induction and host defense against multiple viral infection [43]. In addition, previous studies have shown that DHX9 plays an important role in maintaining gene stability and promotes the survival of tumor cells [44] [45] [46]. Recent proteomic studies also suggest elevated DHX9 expression levels in CRC, whereas DHX36 silence reduced CRC invasiveness and metastasis [26] [47]. These studies indicate that the helicase family may be also involved in the development of CRC. However, the signaling pathways for these molecules are still largely unknown.

Our present study found that the expression of DHX9 increased in clinical CRC samples and/or TCGA database (Figure 5A, C). The expression of DHX36 in CRC was not different in our clinical samples or AOM/DSStreated samples, but increased in ATCG database and was associated with cancer stages (Figure 5D, E, F and Figure 6B). Notably, a recent study found that inhibition of DHX9 promoted death of tumor cells but did not affect the function of normal cells at organ level, indicating that DHX9 may have potential to serve as a safe and effective target for CRC therapy [46]. Interestingly, the expression of Dhxy was decreased in AOM/DSS-treated mice (Figure 5B). The differences between the mouse model and human CRC might be due to the time point difference.

Clinical CRC database indicated that

Figure 4. Correlation between STING signaling-associated molecules expression and cancer stages. The expression data of STING signaling pathway-associated molecules were retrieved from the TCGA microarray database in

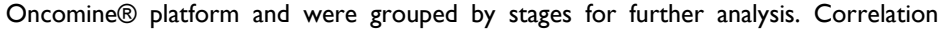
between STING (A), IFII6 (B), DAI(C), DDX4I (D), IRF3 (E), and cancer stages were shown. $\mathrm{n}=22$ for health control, 44 for stage I, 78 for stage II, 52 for stage III, and 23 for stage IV. Data were expressed as mean \pm SEM, Log2 median-centered ratio expression. *p $<0.05$.
DHX9 and DHX36 were upregulated in CRC decreased in clinical CRC, AOM/DSS treated mouse tissues, and TCGA database (Figure 5G, $\mathbf{H}, \mathbf{I})$. In addition, the expression of DDX60 in CRC was associated with cancer stages (Figure tissues. However, the expression of DDX60 
6C). These results consistently indicated that DDX60 were downregulated in the development of CRC.

A previous study showed that DHX9 can recruit MAVS and MyD88 after recognizing dsRNA and CpG modified ssDNA, respectively [48]. Therefore, we analyzed the correlation between DHX9 and MAVS or MyD88 in CRC. We found that DHX9 and MVAS or MyD88 had a positive correlation (Figure 6D, E). On the other hand, DHX36 was reported to interact with DDX1 and DDX21 to form a complex that further signals via TRIF or MyD88 [49]. Our analysis result identified positive correlation between DHX36 and MyD88 but not TRIF (Figure 6F, G).

\section{The expression profile of TLR9 signaling molecules in CRC}

Our previous study showed that the expression of TLR9 in human CRC and AOM/DSS-induced early stage of CRC tissues significantly decreased [35]. Subsequently, we further analyzed the expression profile of TLR9 in clinical CRC samples, AOM/DSS-induced mice, and TCGA database and its adaptor $M y D 88$ in TCGA database in Oncomine platform. No significant change of TLR9 was observed in TCGA database (Figure 7A). No correlation exists between TLR9 expression and cancer stages in Oncomine platform (Figure 7C). The expression of adaptor MyD88 was significant decreased in CRC group (Figure 7B) and associated with cancer stages (Figure 7D). Further analysis showed that a positive correlation existed in TLR9 and MyD88 in CRC tissues (Figure 7E). The overall expression profiles of DNA sensors were summarized (Table 1).
A

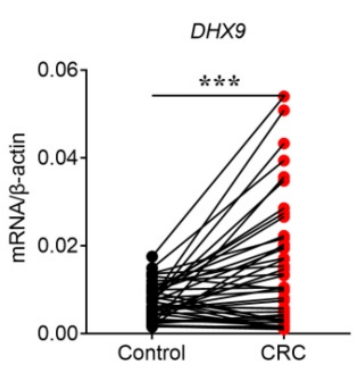

B

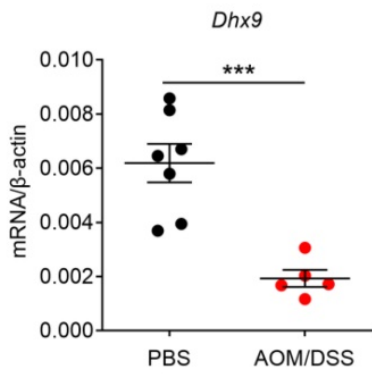

E
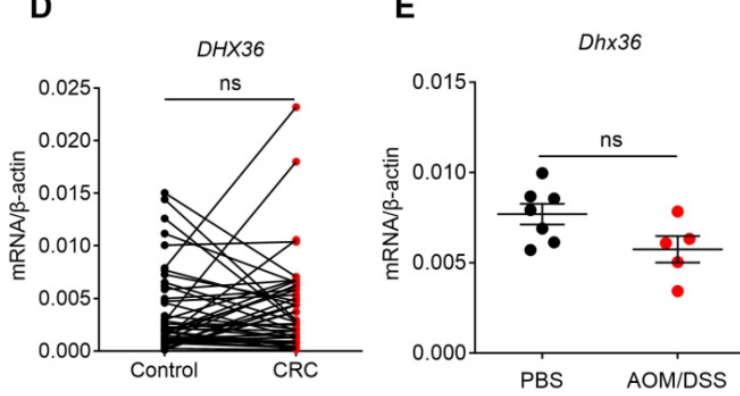

H

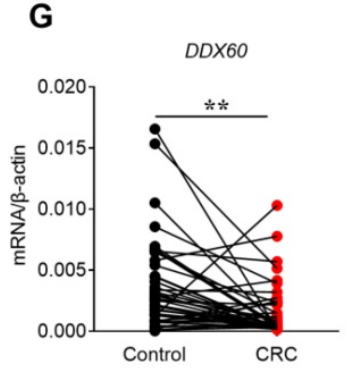

C

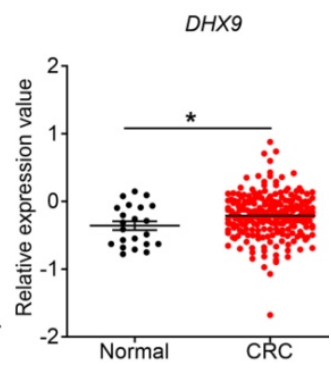

F

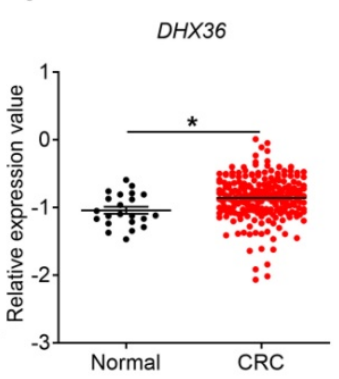

I

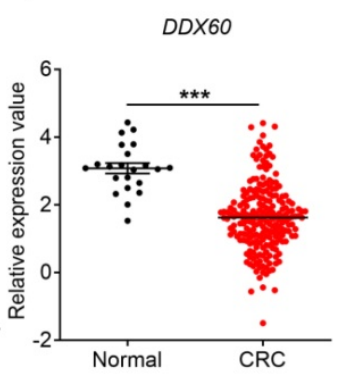

Figure 5. The expression profile of helicases in human and mouse CRC tissues and TCGA database. RNA was extracted from cancer and matched peri-carcinomatous tissues of CRC patients, as well as tissues from AOM/DSS treated mice, and reverse-transcribed into cDNA. Then the gene expression levels of helicases were determined by quantitative PCR. The data from the same patient were connected by straight lines. The data of the expression of helicases were retrieved from the TCGA microarray database in Oncomine ${ }^{\circ}$ platform. Expression of $D H X 9$ (A, B, C), DHX36 (D, E, F), and DDX60 (G, H, I), in human CRC tissues, tissues from AOM/DSS treated mice, and TCGA database. Control: matched peri-carcinomatous tissues, $\mathrm{n}=44$ for clinical samples, data represent two independent experiments; $n=7$ for PBS-treated mice and $n=5$ for AOM/DSS- treated mice. $* p<0.05$, **p $<0.01$, *** $p<0.001$; NS: Not significantly different. 
A

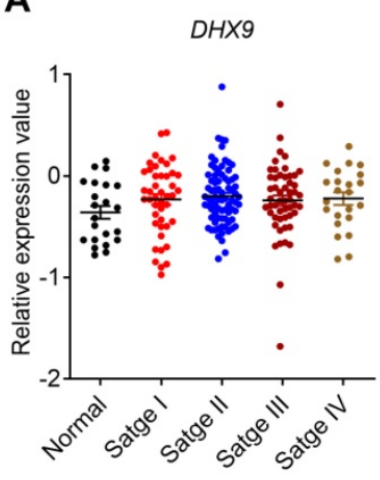

D

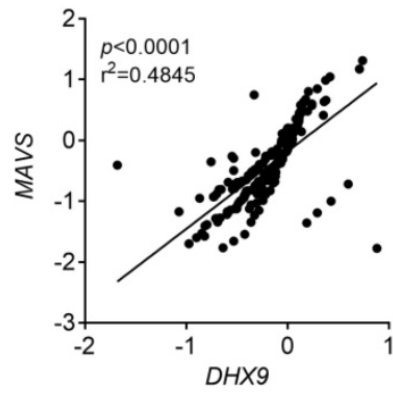

G

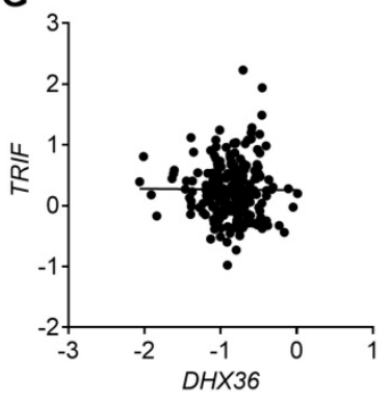

B



E

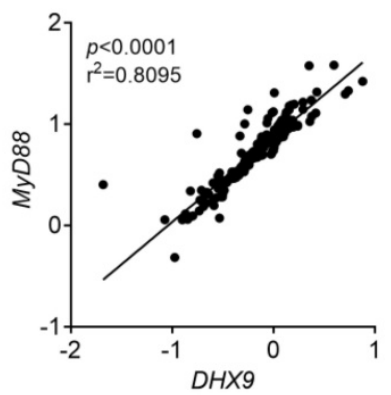

C

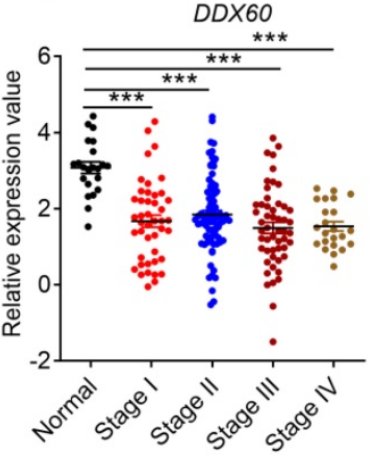

F

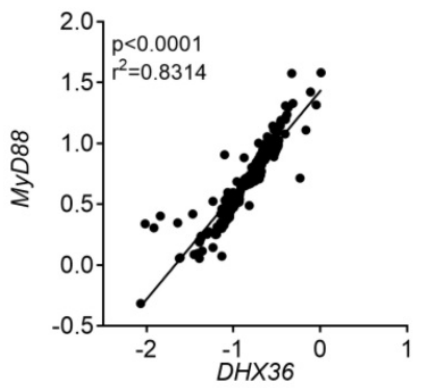

Figure 6. Correlation between helicases expression and cancer stages, and between their expression and MyD88/MAVS/TRIF expression. The expression data of helicases were retrieved from the TCGA microarray database in Oncomine $₫$ platform, and were grouped by stages or patient IDs for further analysis. (A-C) Correlation between DHX9, DHX36, and DDX60 and cancer stages were shown. (D-G) Expression correlation between DHX9 and MAVS/MyD88, DHX36 and MyD88/TRIF. $\mathrm{n}=22$ for health normal control, 44 for stage I, 78 for stage II, 52 for stage III, and 23 for stage IV. Data were expressed as mean \pm SEM, Log2 median-centered ratio expression. $* p<0.05 ; * * * p<0.001$.

Table 1. The summary of expression profiles of DNA sensors and downstream molecules and their correlations with cancer stages in CRC

\begin{tabular}{|c|c|c|c|c|c|}
\hline Genes & & Human CRC tissues & TCGA database & Mouse AOM/DSS treatment & Associated with Cancer stages \\
\hline \multirow[t]{5}{*}{ ALRs } & AIM2 & Downregulated & Downregulated & Downregulated & No \\
\hline & IFI16 & NS & Downregulated & NS & No \\
\hline & TLR9 & Downregulated & NS & Downregulated & No \\
\hline & STING & NS & NS & NS & No \\
\hline & DAI & Downregulated & Downregulated & Downregulated & Yes \\
\hline DDXs & DDX41 & Upregulated & NS & Downregulated & No \\
\hline \multirow[t]{7}{*}{ DHXs } & DHX9 & Upregulated & Upregulated & Downregulated & No \\
\hline & DHX36 & NS & Upregulated & NS & Yes \\
\hline & DDX60 & Downregulated & Downregulated & Downregulated & Yes \\
\hline & MyD88 & / & Downregulated & / & Yes \\
\hline & IRF3 & / & Upregulated & / & Yes \\
\hline & ASC & / & Downregulated & / & Yes \\
\hline & IL-18 & / & Downregulated & / & Yes \\
\hline
\end{tabular}

(Note: NS, not statistically significant) 


\section{The protein levels of DNA sensors in human CRC tissues}

To confirm the gene expression profile, we also tested the protein level of DNA sensors including STING, IFI16, DAI, DDX41, DHX9, DHX36 and DDX60 in CRC and the matched pericarcinomatous tissues using Western-blot analysis. We found that the protein changes of these DNA receptors in CRC were consistent with the gene expression patterns (Figure 8).

\section{Discussion}

Aberrant inflammatory response is an important risk factor of tumor formation, as well as an important feature after tumor formation [50]. In the early stage of tumor formation, inflammatory environment facilitates the activation of various signaling pathways, and the differentiation from normal cell

A

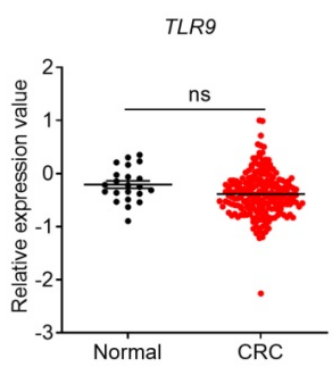

C

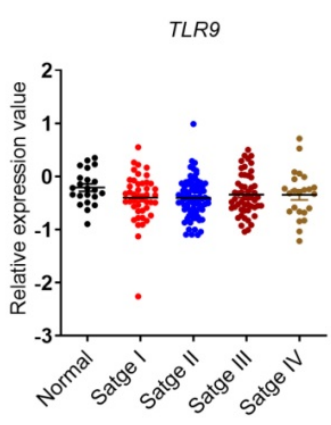

E

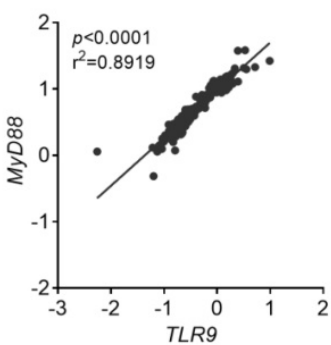

Figure 7. The expression profile of TLR9 in CRC tissues and its correlation with tumor stages or MyD88. The expression data of TLR9 were retrieved from the TCGA microarray database in Oncomine $\AA$ platform and were grouped by stages or patient IDs for further analysis. Expression of TLR9 (A) and MyD88 (B) in TCGA database. Correlation analysis of TLR9(C) and MyD88 (D) in different stages of CRC and health control. (E) Expression correlation between TLR9 and MyD88. $\mathrm{n}=22$ for health normal control, 44 for stage I, 78 for stage II, 52 for stage III, and 23 for stage IV. Data were expressed as mean \pm SEM, Log2 median-centered ratio expression. ${ }^{* * *} p<0.001$; NS: Not significantly different. into hyperplasia toward tumor [50]. In the late stages of tumor formation, tumor cells require a variety of positive or negative regulatory mechanisms to escape immune surveillance [51]. The dysregulation of the immune system compromises the effective host barrier against the formation and development of tumors.

DNA sensors are one of the major sub-family of innate immune receptors that recognize DNA. They are mainly expressed in the cytoplasm of innate immune cells and can be also expressed in some other cells, such as epithelial cells. The activation of these receptors can lead to the elimination of invading pathogenic microorganisms through recognizing exogenous DNA. Subsequent studies have shown that the DNA sensors can also respond to abnormal accumulated endogenous DNA in cytoplasm and is involved in the development of inflammatory diseases and autoimmune diseases [52]. Notably, in recent years, more and more studies have shown that DNA sensors also participate in the development of tumors, although their specific functions and mechanisms are still unknown.

Recent studies suggest that DNA sensors such as AIM2, TLR9, DHX9, DHX36 and adaptor STING may have roles in CRC development, and the other DNA sensors such as DDX41, DDX60, IFI16, and DAI participate in the development of other types of cancers. Here, we firstly highlighted the notion that many DNA sensors may have a regulatory role in the occurrence and development of CRC. However, there is still a lack of systematic understanding on the expression profile, underlying mechanisms and clinical significance of DNA sensors in CRC. Our present study represents a comprehensive analysis of DNA sensor expression in CRC and its relationship with the tumor stages by combining with the clinical CRC samples, mouse CRC samples, and Oncomine platform.

We found that DNA sensors and the downstream molecules, including AIM2, DDX60, TLR9, ASC, MyD88, and IL-18 were down-regulated in CRC. The helicase family members, including DDX41, DHX9, and DHX36, were up-regulated in CRC tissues in comparison with controls. IFI16 and STING were the exception in that they showed no significant change in CRC. Our results of STING and IFI16 were different from some previous studies. Genetic and 
epigenetic difference may exist by regions, racial or the quality of sample. Further analysis showed that the expression of AIM2, DDX41,DHX9, DAI, and DDX60 showed significant differences between controls and CRC samples. Among these DNA sensors, AIM2, DAI, and DDX60 showed consistent change in our clinical CRC samples and TCGA database, and were worthy of further investigation on their role in CRC development and clinical application.

In summary, our present work studied the expression profile of DNA sensors in CRC, which broaden our horizons about the function of DNA sensors beyond immunity against viral infection. Notably, although some agonists of DNA sensors (e.g.
TLR9 and STING) have been applied in clinical or pre-clinical studies and achieved some encouraging outcomes, their effects in different tumors or different stages are largely undefined. Therefore, further understanding on the expression and the role of DNA sensors in different cell types in TME is necessary for rational design of DNA sensor agonists in clinical trials. On the other hand, we noticed that although DHX9, were up-regulated in CRC, they decreased in early stage of AOM/DSS treated mouse. Therefore, the expression of DNA sensors may vary along with diseases progression, and further investigations on their expression patterns in middle and late stages of CRC formation may help to understand the full DNA sensors expression patterns.
A

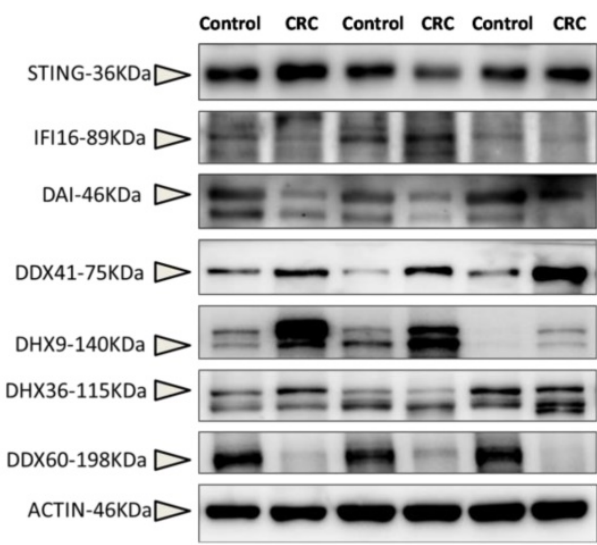

C

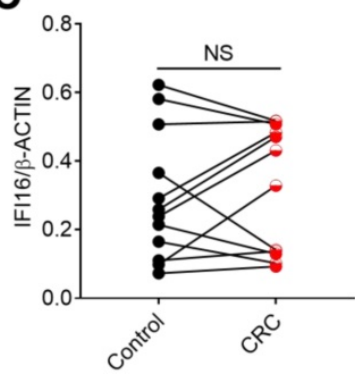

D

G

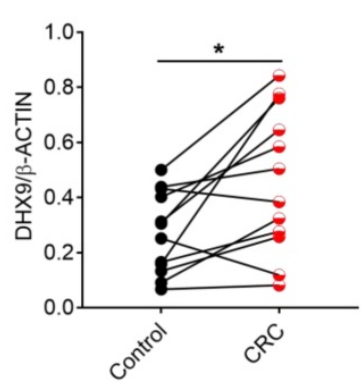

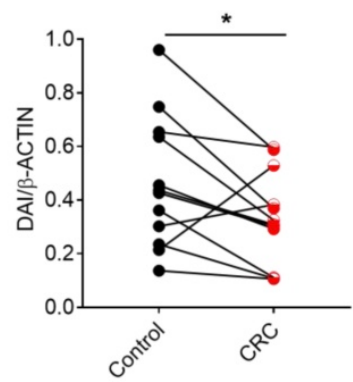

B

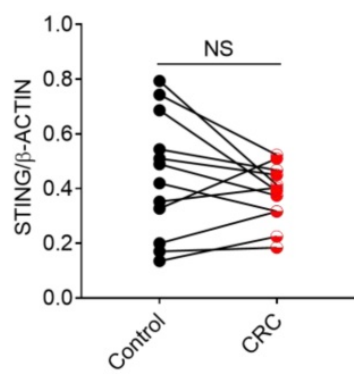

$\mathbf{E}$

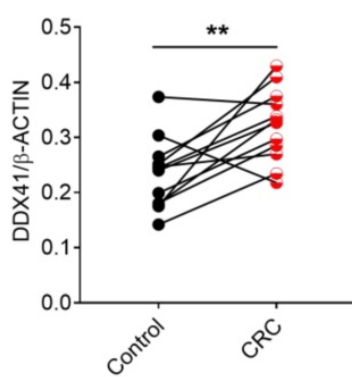

H
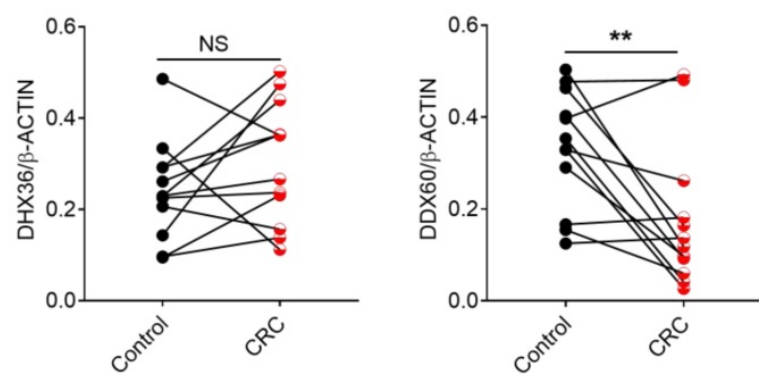

Figure 8. The protein levels of DNA sensors in human CRC tissues. Protein was extracted from cancer and matched peri-carcinomatous tissues of CRC patients, and then the levels of DNA sensors including STING, IFI16, DAI, DDX41, DHX9, DHX36 and DDX60 were determined by Western-blot. (A) The representative Western-blot results. The triangles point to the specific bands of the molecules. Densitometric analysis of band intensity of STING (B), IFI16(C), DAI(D), DDX41(E), DHX9(F), DHX36(G) and DDX60(H) was shown. Control: matched peri-carcinomatous tissues, CRC: colorectal cancer tissues, $\mathrm{n}=12$. * $<$ $0.05 ; *_{p}^{*}<0.01 ;$ NS: Not significantly different. 


\section{Supplementary Material}

Supplementary tables.

http://www.jcancer.org/v11p0893s1.pdf

\section{Acknowledgments}

This work was supported by funds from Talents' Start-up Fund of Gannan Medical University (QD201404), Natural Science Foundation of Jiangxi Province (20151BAB205061, 20171ACB20024, and 20181BAB205032), National Natural Science Foundation of China (31560260, 31960163), and Science and Technology Foundation of Jiangxi Provincial Department of Education (Gjj150937) (All to Zhiping Liu).

\section{Authors' contribution}

Study design: Zhiping Liu; Experiment and statistical analysis: Liangmei $\mathrm{He}$, Yuxia Liu and Weiling Lai; Drafting and editing: Liangmei $\mathrm{He}$, Hongbo Tian, Lingxia Chen, and Lu Xie. All authors read and approved the final manuscript.

\section{Availability of data and materials}

In this study, the TCGA database in the Oncomine platform was chosen to study the expression profile of the DNA sensors (Analysis Type: Cancer vs. Normal Analysis; Cancer Type: Colorectal Cancer; Data Type: mRNA; Sample Type: Clinical Specimen; Genes: every one DNA sensors; Dataset name: TCGA Colorectal, Threshold fold change $\geq 1.5$, $p$ value $\geq$ IE-4, Gene Rank in the top 10). All data generated or analyzed during this study are included in this published article.

\section{Ethics approval and consent to participate}

All the animal experiments were approved by the Animal Ethical and Welfare Committee of Gannan Medical University.

\section{Competing Interests}

The authors have declared that no competing interest exists.

\section{References}

1. Bray F, Ferlay J, Soerjomataram I, Siegel RL, Torre LA, Jemal A. Global cancer statistics 2018: GLOBOCAN estimates of incidence and mortality worldwide for 36 cancers in 185 countries. CA Cancer J Clin. 2018; 68: 394-424.

2. Arnold M, Sierra MS, Laversanne M, Soerjomataram I, Jemal A, Bray F. Global patterns and trends in colorectal cancer incidence and mortality. Gut. 2017; 66: 683-91.

3. Jess T, Rungoe C, Peyrin-Biroulet L. Risk of colorectal cancer in patients with ulcerative colitis: a meta-analysis of population-based cohort studies. Clin Gastroenterol Hepatol. 2012; 10: 639-45.

4. Wang K, Karin M. Tumor-Elicited Inflammation and Colorectal Cancer. Adv Cancer Res. 2015; 128: 173-96.

5. Takeuchi O, Akira S. Pattern recognition receptors and inflammation. Cell. 2010; 140: 805-20.

6. Parlato M, Yeretssian G. NOD-like receptors in intestinal homeostasis and epithelial tissue repair. Int J Mol Sci. 2014; 15: 9594-627.
7. Meylan E, Tschopp J, Karin M. Intracellular pattern recognition receptors in the host response. Nature. 2006; 442: 39-44.

8. He L, Chen Y, Wu Y, Xu Y, Zhang Z, Liu Z. Nucleic acid sensing pattern recognition receptors in the development of colorectal cancer and colitis. Cell Mol Life Sci. 2017; 74: 2395-411.

9. Paludan SR, Bowie AG. Immune sensing of DNA. Immunity. 2013; 38: 870-80.

10. Dempsey A, Bowie AG. Innate immune recognition of DNA: A recent history. Virology. 2015; 479-480: 146-52.

11. Gurtler C, Bowie AG. Innate immune detection of microbial nucleic acids. Trends Microbiol. 2013; 21: 413-20.

12. Li TT, Ogino S, Qian ZR. Toll-like receptor signaling in colorectal cancer: carcinogenesis to cancer therapy. World J Gastroenterol. 2014; 20: 17699-708.

13. Sipos F, Furi I, Constantinovits M, Tulassay Z, Muzes G. Contribution of TLR signaling to the pathogenesis of colitis-associated cancer in inflammatory bowel disease. World J Gastroenterol. 2014; 20: 12713-21.

14. Chen GY. Role of Nlrp6 and Nlrp12 in the maintenance of intestinal homeostasis. Eur J Immunol. 2014; 44: 321-7.

15. Hornung V, Ablasser A, Charrel-Dennis M, Bauernfeind F, Horvath G, Caffrey DR, et al. AIM2 recognizes cytosolic dsDNA and forms a caspase-1-activating inflammasome with ASC. Nature. 2009; 458: 514-8.

16. Wilson JE, Petrucelli AS, Chen L, Koblansky AA, Truax AD, Oyama Y, et al. Inflammasome-independent role of AIM2 in suppressing colon tumorigenesis via DNA-PK and Akt. Nat Med. 2015; 21: 906-13.

17. Man SM, Zhu Q, Zhu L, Liu Z, Karki R, Malik A, et al. Critical Role for the DNA Sensor AIM2 in Stem Cell Proliferation and Cancer. Cell. 2015; 162: 45-58

18. Chen J, Wang Z, Yu S. AIM2 regulates viability and apoptosis in human colorectal cancer cells via the PI3K/Akt pathway. Onco Targets Ther. 2017; 10: 811-7.

19. Ishikawa H, Ma Z, Barber GN. STING regulates intracellular DNA-mediated, type I interferon-dependent innate immunity. Nature. 2009; 461: 788-92.

20. Zhang Z, Yuan B, Bao M, Lu N, Kim T, Liu YJ. The helicase DDX41 senses intracellular DNA mediated by the adaptor STING in dendritic cells. Nat Immunol. 2011; 12: 959-65.

21. Sun L, Wu J, Du F, Chen X, Chen ZJ. Cyclic GMP-AMP synthase is a cytosolic DNA sensor that activates the type I interferon pathway. Science. 2013; 339: 786-91.

22. Unterholzner L, Keating SE, Baran M, Horan KA, Jensen SB, Sharma S, et al. IFI16 is an innate immune sensor for intracellular DNA. Nat Immunol. 2010; 11: $997-1004$.

23. Zhu Q, Man SM, Gurung P, Liu Z, Vogel P, Lamkanfi M, et al. Cutting edge: STING mediates protection against colorectal tumorigenesis by governing the magnitude of intestinal inflammation. J Immunol. 2014; 193: 4779-82.

24. Ahn J, Konno H, Barber GN. Diverse roles of STING-dependent signaling on the development of cancer. Oncogene. 2015; 34: 5302-8.

25. He L, Xiao X, Yang X, Zhang Z, Wu L, Liu Z. STING signaling in tumorigenesis and cancer therapy: A friend or foe? Cancer Lett. 2017; 402: 203-12.

26. Albrethsen J, Knol JC, Piersma SR, Pham TV, de Wit M, Mongera S, et al. Subnuclear proteomics in colorectal cancer: identification of proteins enriched in the nuclear matrix fraction and regulation in adenoma to carcinoma progression. Mol Cell Proteomics. 2010; 9: 988-1005.

27. Jiang Y, Zhu Y, Liu ZJ, Ouyang S. The emerging roles of the DDX41 protein in immunity and diseases. Protein Cell. 2017; 8: 83-9.

28. Fu TY, Wu CN, Sie HC, Cheng JT, Lin YS, Liou HH, et al. Subsite-specific association of DEAD box RNA helicase DDX60 with the development and prognosis of oral squamous cell carcinoma. Oncotarget. 2016; 7: 85097-108.

29. Rhodes DR, Kalyana-Sundaram S, Mahavisno V, Varambally R, Yu J, Briggs $\mathrm{BB}$, et al. Oncomine 3.0: genes, pathways, and networks in a collection of 18,000 cancer gene expression profiles. Neoplasia. 2007; 9: 166-80.

30. Snider AJ, Bialkowska AB, Ghaleb AM, Yang VW, Obeid LM, Hannun YA. Murine Model for Colitis-Associated Cancer of the Colon. Methods Mol Biol. 2016; 1438: 245-54.

31. Sharma D, Malik A, Guy CS, Karki R, Vogel P, Kanneganti TD. Pyrin Inflammasome Regulates Tight Junction Integrity to Restrict Colitis and Tumorigenesis. Gastroenterology. 2018; 154: 948-64 e8.

32. Karki R, Man SM, Malireddi RK, Kesavardhana S, Zhu Q, Burton AR, et al. NLRC3 is an inhibitory sensor of PI3K-mTOR pathways in cancer. Nature. 2016.

33. Zaki MH, Vogel P, Malireddi RK, Body-Malapel M, Anand PK, Bertin J, et al. The NOD-like receptor NLRP12 attenuates colon inflammation and tumorigenesis. Cancer Cell. 2011; 20: 649-60.

34. Dihlmann S, Tao S, Echterdiek F, Herpel E, Jansen L, Chang-Claude J, et al. Lack of Absent in Melanoma 2 (AIM2) expression in tumor cells is closely associated with poor survival in colorectal cancer patients. Int J Cancer. 2014; 135: 2387-96

35. Becker C, Weigel D. Epigenetic variation: origin and transgenerational inheritance. Curr Opin Plant Biol. 2012; 15: 562-7.

36. Liu R, Truax AD, Chen L, Hu P, Li Z, Chen J, et al. Expression profile of innate immune receptors, NLRs and AIM2, in human colorectal cancer: correlation with cancer stages and inflammasome components. Oncotarget. 2015; 6: 33456-69.

37. HE L, Chen Y, Wu Y, Liu Y, Zhang Z, Liu Z. Expression profle of Toll-like receptors 9 in colorectal cancer. Journal of Gannan Medical University. 2017; 37: $32-7$ 
38. Woo SR, Fuertes MB, Corrales L, Spranger S, Furdyna MJ, Leung MY, et al. STING-dependent cytosolic DNA sensing mediates innate immune recognition of immunogenic tumors. Immunity. 2014; 41: 830-42.

39. Deng L, Liang H, Xu M, Yang X, Burnette B, Arina A, et al. STING-Dependent Cytosolic DNA Sensing Promotes Radiation-Induced Type I Interferon-Dependent Antitumor Immunity in Immunogenic Tumors. Immunity. 2014; 41: 843-52.

40. Burdette DL, Monroe KM, Sotelo-Troha K, Iwig JS, Eckert B, Hyodo M, et al. STING is a direct innate immune sensor of cyclic di-GMP. Nature. 2011; 478: 515-8.

41. Xia T, Konno H, Ahn J, Barber GN. Deregulation of STING Signaling in Colorectal Carcinoma Constrains DNA Damage Responses and Correlates With Tumorigenesis. Cell Rep. 2016; 14: 282-97.

42. Yang CA, Huang HY, Chang YS, Lin CL, Lai IL, Chang JG. DNA-Sensing and Nuclease Gene Expressions as Markers for Colorectal Cancer Progression. Oncology. 2017; 92: 115-24.

43. Fullam A, Schroder M. DExD/H-box RNA helicases as mediators of anti-viral innate immunity and essential host factors for viral replication. Biochim Biophys Acta. 2013; 1829: 854-65.

44. Jain A, Bacolla A, Del Mundo IM, Zhao J, Wang G, Vasquez KM. DHX9 helicase is involved in preventing genomic instability induced by alternatively structured DNA in human cells. Nucleic Acids Res. 2013; 41: 10345-57.

45. Fidaleo M, Svetoni F, Volpe E, Minana B, Caporossi D, Paronetto MP. Genotoxic stress inhibits Ewing sarcoma cell growth by modulating alternative pre-mRNA processing of the RNA helicase DHX9. Oncotarget. 2015; 6: 31740-57.

46. Lee T, Paquet M, Larsson O, Pelletier J. Tumor cell survival dependence on the DHX9 DExH-box helicase. Oncogene. 2016; 35: 5093-105.

47. Matsumura K, Kawasaki Y, Miyamoto M, Kamoshida Y, Nakamura J, Negishi $\mathrm{L}$, et al. The novel G-quadruplex-containing long non-coding RNA GSEC antagonizes DHX36 and modulates colon cancer cell migration. Oncogene. 2017; 36: 1191-9.

48. Hornung V. SnapShot: Nucleic acid immune sensors, part 2. Immunity. 2014; 41: 1066- e1.

49. Zhang Z, Kim T, Bao M, Facchinetti V, Jung SY, Ghaffari AA, et al. DDX1, DDX21, and DHX36 helicases form a complex with the adaptor molecule TRIF to sense dsRNA in dendritic cells. Immunity. 2011; 34: 866-78.

50. Kundu JK, Surh YJ. Inflammation: gearing the journey to cancer. Mutat Res. 2008; 659: 15-30

51. Ribatti D. The concept of immune surveillance against tumors. The first theories. Oncotarget. 2017; 8: 7175-80.

52. Kawasaki T, Kawai T, Akira S. Recognition of nucleic acids by pattern-recognition receptors and its relevance in autoimmunity. Immunol Rev. 2011; 243: 61-73 